\title{
Randomized Distributed Decision
}

\author{
Pierre Fraigniaud \\ Amos Korman \\ CNRS and University Paris Diderot, France. \{pierre.fraigniaud,amos.korman\}@liafa.jussieu.fr* \\ Merav Parter David Peleg \\ The Weizmann Institute of Science, Rehovot, Israel. \{merav.parter,david.peleg\}@weizmann.ac.il ${ }^{\dagger}$
}

\begin{abstract}
The paper tackles the power of randomization in the context of locality by analyzing the ability to "boost" the success probability of deciding a distributed language. The main outcome of this analysis is that the distributed computing setting contrasts significantly with the sequential one as far as randomization is concerned. Indeed, we prove that in some cases, the ability to increase the success probability for deciding distributed languages is rather limited.

Informally, a $(p, q)$-decider for a language $\mathcal{L}$ is a distributed randomized algorithm which accepts instances in $\mathcal{L}$ with probability at least $p$ and rejects instances outside of $\mathcal{L}$ with probability at least $q$. It is known that every hereditary language that can be decided in $t$ rounds by a $(p, q)$-decider, where $p^{2}+q>1$, can actually be decided deterministically in $O(t)$ rounds. In one of our results we give evidence supporting the conjecture that the above statement holds for all distributed languages and not only for hereditary ones. This is achieved by considering the restricted case of path topologies.

We then turn our attention to the range below the aforementioned threshold, namely, the case where $p^{2}+q \leq 1$. For $k \in \mathbb{N}^{*} \cup\{\infty\}$, we define the class $B_{k}(t)$ to be the set of all languages decidable in at most $t$ rounds by a $(p, q)$-decider, where $p^{1+\frac{1}{k}}+q>1$. It is easy to see that every language is decidable (in zero rounds) by a $(p, q)$-decider satisfying $p+q=1$. Hence, the hierarchy $B_{k}$ provides a spectrum of complexity classes between determinism ( $k=1$, under the above conjecture) and complete randomization $(k=\infty)$. We prove that all these classes are separated, in a strong sense: for every integer $k \geq 1$, there exists a language $\mathcal{L}$ satisfying $\mathcal{L} \in B_{k+1}(0)$ but $\mathcal{L} \notin B_{k}(t)$ for any $t=o(n)$. In addition, we show that $B_{\infty}(t)$ does not contain all languages, for any $t=o(n)$. In other words, we obtain the following hierarchy:

$$
B_{1}(t) \subset B_{2}(t) \subset \cdots \subset B_{\infty}(t) \subset \text { All . }
$$
\end{abstract}

Finally, we show that if the inputs can be restricted in certain ways, then the ability to boost the success probability becomes almost null, and, in particular, derandomization is not possible even beyond the threshold $p^{2}+q=1$.

All our results hold with respect to the $\mathcal{L O C} \mathcal{A L}$ model of computation as well as with respect to the

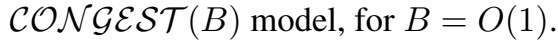

\section{Introduction}

\subsection{Background and Motivation}

The impact of randomization on computation is one of the most central questions in computer science. In particular, in the context of distributed computing, the question of whether randomization helps in improving locality for construction problems has been studied extensively. While most of these studies were

\footnotetext{
* Supported by the ANR projects DISPLEXITY and PROSE, and by the INRIA project GANG.

${ }^{\dagger}$ Supported in part by the Israel Science Foundation (grant 894/09), the US-Israel Binational Science Foundation (grant 2008348), the Israel Ministry of Science and Technology (infrastructures grant), and the Citi Foundation.
} 
problem-specific, several attempts have been made for tackling this question from a more general and unified perspective. For example, Naor and Stockmeyer [26] focus on a class of problems called LCL (essentially a subclass of the class LD discussed below), and show that if there exists a randomized algorithm that constructs a solution for a problem in LCL in a constant number of rounds, then there is also a constant time deterministic algorithm constructing a solution for that problem.

Recently, this question has been studied in the context of local decision, where one aims at deciding locally whether a given global input instance belongs to some specified language [13]. The localities of deterministic algorithms and randomized Monte Carlo algorithms are compared in [13], in the $\mathcal{L O C} \mathcal{A L}$ model (cf. [28]). One of the main results of [13] is that randomization does not help for locally deciding hereditary languages if the success probability is beyond a certain guarantee threshold. More specifically, a $(p, q)$-decider for a language $\mathcal{L}$ is a distributed randomized Monte Carlo algorithm that accepts instances in $\mathcal{L}$ with probability at least $p$ and rejects instances outside of $\mathcal{L}$ with probability at least $q$. It was shown in [13] that every hereditary language that can be decided in $t$ rounds by a $(p, q)$-decider, where $p^{2}+$ $q>1$, can actually be decided deterministically in $O(t)$ rounds. On the other hand, [13] showed that the aforementioned threshold is sharp, at least when hereditary languages are concerned. In particular, for every $p$ and $q$, where $p^{2}+q \leq 1$, there exists an heredirtary language that cannot be decided deterministically in $o(n)$ rounds, but can be decided in zero number of rounds by a $(p, q)$-decider.

In one of our results we provide evidence supporting the conjecture that the above statement holds for all distributed languages and not only for hereditary ones. This is achieved by considering the restricted case of path topologies. In addition, we present a more refined analysis for the family of languages that can be decided randomly but not deterministically. That is, we focus on the family of languages that can be decided locally by a $(p, q)$-decider, where $p^{2}+q \leq 1$, and introduce an infinite hierarchy of classes within this family, characterized by the specific relationships between the parameters $p$ and $q$. As we shall see, our results imply that the distributed computing setting contrasts significantly with the sequential one as far as randomization is concerned. Indeed, we prove that in some cases, the ability to increase the success probability for deciding distributed languages is very limited.

\subsection{Model}

We consider the $\mathcal{L O C} \mathcal{A} \mathcal{L}$ model (cf. [28]), which is a standard distributed computing model capturing the essence of spatial locality. In this model, processors are woken up simultaneously, and computation proceeds in fault-free synchronous rounds during which every processor exchanges messages of unlimited size with its neighbors, and performs arbitrary computations on its data. It is important to stress that all the algorithmic constructions that we employ in our positive results use messages of constant size (some of which do not use any communication at all). Hence, all our results apply not only to the $\mathcal{L} \mathcal{O C} \mathcal{A} \mathcal{L}$ model of computation but also to more restricted models, for example, the $\mathcal{C O N G \mathcal { G S T }}(B)$ mode $\left.\right|^{1}$, where $B=O(1)$.

A distributed algorithm $\mathcal{A}$ that runs on a graph $G$ operates separately on each connected component of $G$, and nodes of a component $C$ of $G$ cannot distinguish the underlying graph $G$ from $C$. For this reason, we consider connected graphs only.

We focus on distributed decision tasks. Such a task is characterized by a finite or infinite set $\Sigma$ of symbols (e.g., $\Sigma=\{0,1\}$, or $\Sigma=\{0,1\}^{*}$ ), and by a distributed language $\mathcal{L}$ defined on this set of symbols (see below). An instance of a distributed decision task is a pair $(G, \mathbf{x})$ where $G$ is an $n$-node connected graph, and $\mathbf{x} \in \Sigma^{n}$, that is, every node $v \in V(G)$ is assigned as its local input a value $\mathbf{x}(v) \in \Sigma$. (In some cases, the local input of every node is empty, i.e., $\Sigma=\{\epsilon\}$, where $\epsilon$ denotes the empty binary string.) We define a distributed language as a decidable collection $\mathcal{L}$ of instances ${ }^{2}$

\footnotetext{
${ }^{1}$ Essentially, the $\mathcal{C O} \mathcal{N G} \mathcal{E S T}(B)$ model is similar to the $\mathcal{L O C} \mathcal{A} \mathcal{L}$ model, except that the message size is assumed to be bounded by at most $B$ bits (for more details, see [28]).

${ }^{2}$ Note that an undecidable collection of instances remains undecidable in the distributed setting too.
} 
In the context of distributed computing, each processor must produce a boolean output, and the decision is defined by the conjunction of the processors outputs, i.e., if the instance belongs to the language, then all processors must output "yes", and otherwise, at least one processor must output "no". Formally, for a distributed language $\mathcal{L}$, we say that a distributed algorithm $\mathcal{A}$ decides $\mathcal{L}$ if and only if for every instance $(G, \mathbf{x})$ and id-assignment Id, every node $v$ of $G$ eventually terminates and produces an output denoted out $_{\mathcal{A}}(G, \mathbf{x}, \mathrm{Id}, v)$, which is either "yes" or "no", satisfying the following decision rules:

- If $(G, \mathbf{x}) \in \mathcal{L}$ then $\operatorname{out}_{\mathcal{A}}(G, \mathbf{x}, \operatorname{Id}, v)=$ "yes" for every node $v \in V(G)$;

- If $(G, \mathbf{x}) \notin \mathcal{L}$ then out $_{\mathcal{A}}(G, \mathbf{x}, \operatorname{Id}, v)=$ "no" for at least one node $v \in V(G)$.

Observe that decision problems provide a natural framework for tackling fault-tolerance: the processors have to collectively check whether the network is fault-free, and a node detecting a fault raises an alarm. In fact, many natural problems can be phrased as decision problems, for example: "is the network planar?" or "is there a unique leader in the network?". Moreover, decision problems occur naturally when one aims at checking the validity of the output of a computational task, such as "is the produced coloring legal?", or "is the constructed subgraph an MST?".

The class of decision problems that can be solved in at most $t$ communication rounds is denoted by $\mathrm{LD}(t)$, for local decision. More precisely, let $t$ be a function of triplets $(G, \mathbf{x}, \mathrm{Id})$, where Id denotes the identity assignment to the nodes of $G$. Then $\operatorname{LD}(t)$ is the class of all distributed languages that can be decided by a distributed algorithm that runs in at most $t$ communication rounds. The randomized (Monte Carlo 2-sided error) version of the class $\operatorname{LD}(t)$ is denoted $\operatorname{BPLD}(t, p, q)$, which stands for bounded-error probabilistic local decision, and provides an analog of BPP for distributed computing, where $p$ and $q$ respectively denote the yes-error and the no-error guarantees. More precisely, a randomized distributed algorithm is a distributed algorithm $\mathcal{A}$ that enables every node $v$, at any round $r$ during its execution, to generate a certain number of random bits. For constants $p, q \in(0,1]$, we say that a randomized distributed algorithm $\mathcal{A}$ is a $(p, q)$-decider for $\mathcal{L}$, or, that it decides $\mathcal{L}$ with "yes" success probability $p$ and "no" success probability $q$, if and only if for every instance $(G, \mathbf{x})$ and id-assignment Id, every node of $G$ eventually terminates and outputs "yes" or "no", and the following properties are satisfied:

- If $(G, \mathbf{x}) \in \mathcal{L}$ then $\operatorname{Pr}[\forall v \in V(G)$, out $_{\mathcal{A}}(G, \mathbf{x}, \mathrm{Id}, v)=$ "yes"] $\geq p$

- If $(G, \mathbf{x}) \notin \mathcal{L}$ then $\operatorname{Pr}[\exists v \in V(G)$, out $_{\mathcal{A}}(G, \mathbf{x}, \mathrm{Id}, v)=$ "no"] $\geq q$.

The probabilities in the above definition are taken over all possible coin tosses performed by the nodes. The running time of a $(p, q)$-decider executed on a node $v$ depends on the triple $(G, \mathbf{x}, \mathrm{Id})$ and on the results of the coin tosses. In the context of a randomized algorithm, $T_{v}(G, \mathbf{x}, \mathrm{Id})$ denotes the maximal running time of the algorithm on $v$ over all possible coin tosses, for the instance $(G, \mathbf{x})$ and id-assignment Id. Now, just as in the deterministic case, the running time $T$ of the $(p, q)$-decider is the maximum running time over all nodes. Note that by definition of the distributed Monte-Carlo algorithm, both $T_{v}$ and $T$ are deterministic. For constant $p, q \in(0,1]$ and a function $t$ of triplets $(G, \mathbf{x}, \operatorname{Id}), \operatorname{BPLD}(t, p, q)$ is the class of all distributed languages that have a randomized distributed $(p, q)$-decider running in time at most $t$ (i.e., can be decided in time at most $t$ by a randomized distributed algorithm with "yes" success probability $p$ and "no"success probability $q$ ).

Our main interest within this context is in studying the connections between the classes $\operatorname{BPLD}(t, p, q)$. In particular, we are interested in the question of whether one can "boost" the success probabilities of a $(p, q)$ decider. (Recall that in the sequential Monte Carlo setting, such "boosting" can easily be achieved by repeating the execution of the algorithm a large number of times.) Our starting point is the recent result of [13] that, for the class of hereditary languages (i.e., closed under sub-graphs), the relation $p^{2}+q=1$ is a sharp threshold for randomization. That is, for hereditary languages, $\bigcup_{p^{2}+q>1} \operatorname{BPLD}(t, p, q)$ collapses to $\operatorname{LD}(O(t))$, but 
for any $p, q \in(0,1]$ such that $p^{2}+q \leq 1$ there exists a language $\mathcal{L} \in \operatorname{BPLD}(0, p, q)$, while $\mathcal{L} \notin \operatorname{LD}(t)$ for any $t=o(n)$. We conjecture that the hereditary assumption can be removed and we give some evidence supporting this conjecture. Aiming at analyzing the collection of classes $\bigcup_{p^{2}+q \leq 1} \operatorname{BPLD}(t, p, q)$, we consider the following set of classes:

$$
B_{k}(t)=\bigcup_{p^{1+1 / k}+q>1} \operatorname{BPLD}(t, p, q)
$$

for any positive integer $k$, as well as the class

$$
B_{\infty}(t)=\bigcup_{p+q>1} \operatorname{BPLD}(t, p, q) .
$$

Hence, our conjecture states that $B_{1}(t)=\mathrm{LD}(O(t))$. Note that the class $\bigcup_{p+q \geq 1} \operatorname{BPLD}(0, p, q)$ contains all languages, using a $(1,0)$-decider that systematically returns "yes" at every node (without any communication). Hence, the classes $B_{k}$ provide a smooth spectrum of randomized distributed complexity classes, from the class of deterministically decidable languages (under our conjecture) to the class of all languages. The ability of boosting the success probabilities of a $(p, q)$-decider is directly related to the question of whether these classes are different, and to what extent.

\subsection{Our results}

One of the main outcomes of this paper is a proof that boosting success probabilities in the distributed setting appears to be quite limited. By definition, $B_{k}(t) \subseteq B_{k+1}(t)$ for any $k$ and $t$. We prove that these inclusions are strict. In fact, our separation result is much stronger. We prove that there exists a language in $B_{k+1}(0)$ that is not in $B_{k}(t)$ for any $t=o(n)$. Moreover, we prove that Tree $\notin B_{\infty}(t)$ for any $t=o(n)$, where Tree $=\{(G, \epsilon): G$ is a tree $\}$. Hence, $B_{\infty}(t)$ does not contain all languages, even for $t=o(n)$. In summary, we obtain the following hierarchy.

$$
B_{1}(t) \subset B_{2}(t) \subset \cdots \subset B_{\infty}(t) \subset \text { All } .
$$

These results demonstrate that boosting the probability of success might be doable, but only from a $(p, q)$ pair satisfying $p^{1+1 /(k+1)}+q>1$ to a $(p, q)$ pair satisfying $p^{1+1 / k}+q>1$ (with the extremes excluded). It is an open question whether $B_{k+1}(t)$ actually collapses to $\operatorname{BPLD}(O(t), p, q)$, where $p^{1+1 / k}+q=1$, or whether there exist intermediate classes.

Recall that every hereditary language in $B_{1}(t)$ is also in $\operatorname{LD}(O(t))$ [13]. We conjecture that this derandomization result holds for all languages and not only for hereditary ones. We give evidence supporting this conjecture by showing that restricted to path topologies, finite input and constant running time $t$, the statement $B_{1}(t) \subseteq \mathrm{LD}(O(t))$ holds without assuming the hereditary property. This evidence seems to be quite meaningful especially since all our separation results hold even if we restrict ourselves to decision problems on path topologies.

Finally, we show that the situation changes drastically if the distribution of inputs can be restricted in certain ways. Indeed, we show that for every two reals $0<r<r^{\prime}$, there exists a language in $C_{r^{\prime}}(0)$ that is not in $C_{r}(t)$ for any $t=o(n)$, where the $C$-classes are the extension of the $B$-classes to decision problems in which the inputs can be restricted.

All our results hold not only with respect to the $\mathcal{L O C} \mathcal{A L}$ model but also with respect to more restrictive

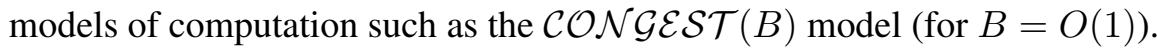




\subsection{Related work}

The notion of local decision and local verification of languages has received quite a lot of attention recently. In the $\mathcal{L O C} \mathcal{A} \mathcal{L}$ model, for example, solving a decision problem requires the processors to independently inspect their local neighborhood and collectively decide whether the global instance belongs to some specified language. Inspired by classical computation complexity theory, Fraigniaud et al[13] suggested that the study of decision problems may lead to new structural insights also in the more complex distributed computing setting. Indeed, following that paper, efforts were made to form a fundamental computational complexity theory for distributed decision problems in various other aspects of distributed computing [13, 14, 15, 16].

The classes LD, NLD and BPLD defined in [13] are the distributed analogues of the classes P, NP and BPP, respectively. The contribution of [13] is threefold: it establishes the impact of nondeterminism, randomization, and randomization + nondeterminism, on local computation. This is done by proving structural results, developing a notion of local reduction and establishing completeness results. One of the main results is the existence of a sharp threshold for randomization, above which randomization does not help (at least for hereditary languages). More precisely the BPLD classes were classified into two: below and above the randomization threshold. The current paper "zooms" into the spectrum of classes below the randomization threshold, and defines a hierarchy of an infinite set of BPLD classes, each of which is separated from the class above it in the hierarchy.

The question of whether randomization helps in improving locality for construction problems has been studied extensively. Naor and Stockmeyer [26] considered a subclass of $\operatorname{LD}(O(1))$, called LCL ${ }^{3}$, and studied the question of how to compute in $O(1)$ rounds the constructive versions of decision problems in LCL. The paper demonstrates that randomization does not help, in the sense that if a problem has a local Monte Carlo randomized algorithm, then it also has a local deterministic algorithm. There are several differences between the setting of [26] and ours. First, [26] considers the power of randomization for constructing a solution, whereas we study the power of randomization for deciding languages 4 Second, while [26] deals with constant time computations, our separation results apply to arbitrary time computations, potentially depending on the size of the instance (graph and input). To summarize, the different settings imply different impacts for randomization: while this current paper as well as [13] show that randomization can indeed help for improving locality of decision problems, [26] shows that when it comes to constructing a solution for a problem in LCL in constant time, randomization does not help. The question of whether randomization helps for constructing solutions to some specific problems in localized computational models such as MIS, $(\Delta+$ 1)-coloring, and maximal matching has been also studied in [2, 5, 23, 24, 25, 27, 29]. The original theoretical basis for nondeterminism in local computation was laid by the theories of proof-labeling schemes [17, 19. 20, 21], which resemble the notion of NLD, and local computation with advice [7, 10, 11, 12]. These notions also bear some similarities to the notions of local detection [1], local checking [4], or silent stabilization [9], which were introduced in the context of self-stabilization [8]. In addition, NLD seems to be related also to the theory of lifts [3].

Finally, the classification of decision problems in distributed computing has been studied in several other

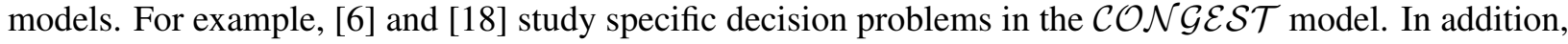
decision problems have been studied in the asynchrony discipline too, specifically in the framework of waitfree computation [15, 16] and mobile agents computing [14]. In the wait-free model, the main issues are not spatial constraints but timing constraints (asynchronism and faults). The main focus of [16] is deterministic protocols aiming at studying the power of the "decoder", i.e., the interpretation of the results. While this

\footnotetext{
${ }^{3} \mathrm{LCL}$ is essentially $\mathrm{LD}(O(1))$ restricted to languages involving graphs of constant maximum degree and processor inputs taken from a set of constant size.

${ }^{4}$ There is a fundamental difference between such tasks when locality is concerned. Indeed, whereas the validity of constructing a problem in LCL is local (by definition), the validity in our setting is "global", in the sense that in an illegal instance, it is sufficient that at least one vertex in the entire network outputs "no".
} 
paper essentially considers the AND-checker, (as a global "yes" corresponds to all processes saying "yes"), [16] deals with other interpretations, including more values (not only "yes" and "no"), with the objective of designing checkers that use the smallest number of values.

\section{Preliminaries}

This section recalls some previous results from the literature, to be used throughout in the paper. Let us

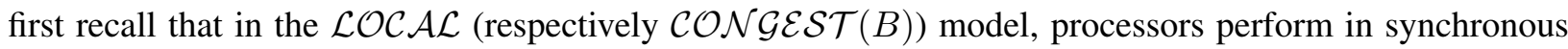
rounds, and, in each round, every processor (1) sends messages of arbitrary (resp., $O(B)$ bits) size to its neighbors, (2) receives messages from its neighbors, and (3) performs arbitrary individual computations. After a number of rounds (that may depend on the network $G$ connecting the processors, and may vary among the processors, since nodes have different identities, potentially different inputs, and are typically located at non-isomorphic positions in the network), every processor $v$ terminates and generates its output.

Consider a distributed $(p, q)$-decider $\mathcal{A}$ running in a network $G$ with input $\mathbf{x}$ and identity assignment Id (assigning distinct integers to the nodes of $G$ ). The output of processor $v$ in this scenario is denoted by out $_{\mathcal{A}}(G, \mathbf{x}, \mathrm{Id}, v)$, or simply out $(v)$ when the parameters are clear from the context. In the case of decision problem, out $(v) \in\{$ “yes", "no" $\}$ for every processor $v$.

An $n$-node path $P$ is represented as a sequence $P=(1, \ldots, n)$, oriented from left to right. (However, node $i$ does not know its position in the path.) Given an instance $(P, \mathbf{x})$ with ID's Id and a subpath $S \subset$ $P$, let $\mathbf{x}_{S}$ (respectively $\operatorname{Id}_{S}$ ) be the restriction of $\mathbf{x}$ (resp., Id) to $S$. We sometimes refer to subpath $S=$ $\left(u_{i}, \ldots, u_{j}\right) \subset P$ as $S=[i, j]$. For a set $U \subseteq V(G)$, let $\mathcal{E}(G, \mathbf{x}, \mathrm{Id}, U)$ denote the event that, when running $\mathcal{A}$ on $(G, \mathbf{x})$ with id-assignment Id, all nodes in $U$ output "yes". Given a language $\mathcal{L}$, an instance $(G, \mathbf{x})$ is called legal iff $(G, \mathbf{x}) \in \mathcal{L}$.

Given a time bound $t$, a subpath $S=[i, j]$ is called an internal subpath of $P$ if $i \geq t+2$ and $j \leq n-t-1$. Note that if the subpath $S$ is internal to $P$, then when running a $t$-round algorithm, none of the nodes in $S$ "sees" the endpoints of $P$.

We now define an important concept, which is crucial in the proofs of our separation results.

Definition 2.1 Let $S$ be a subpath of $P$. For $\delta \in[0,1], S$ is said to be a $(\delta, \lambda)$-secure subpath if $|S| \geq \lambda$ and $\operatorname{Pr}[\mathcal{E}(P, \mathbf{x}, \operatorname{Id}, V(S))] \geq 1-\delta$.

We typically use $(\delta, \lambda)$-secure subpaths for values of $\lambda \geq 2 t+1$ where $t$ is the running time of the $(p, q)$-decider $\mathcal{A}$ on $(P, \mathbf{x})$ for some fixed identity assignment Id. Indeed, it is known [13] that if $(P, \mathbf{x}) \in \mathcal{L}$, then every long enough subpath $S$ of $P$ contains an internal $(\delta, \lambda)$-secure subpath $S^{\prime}$. More precisely, define

$$
\ell(\delta, \lambda)=4(\lambda+2 t)\lceil\log p / \log (1-\delta)\rceil
$$

We have the following:

Fact $2.2([\mathbf{1 3}])$ Let $(P, \mathbf{x}) \in \mathcal{L}, \delta \in[0,1], \lambda \geq 1$. Then for every $\ell(\delta, \lambda)$-length subpath $S$ there is a subpath $S^{\prime}$ (internal to $S$ ) that is $(\delta, \lambda)$-secure.

For completeness, we provide the proof of this fact in the Appendix. To avoid cumbersome notation, when $\lambda=2 t+1$, we may omit it and refer to $(\delta, 2 t+1)$-secure subpaths as $\delta$-secure subpaths. In addition, set

$$
\ell(\delta):=\ell(\delta, 2 t+1)
$$

Let us next illustrate a typical use of Fact 2.2. Recall that $t$ denotes the running time of the $(p, q)$-decider $\mathcal{A}$ on $(P, \mathbf{x}) \in \mathcal{L}$ with IDs Id. Let $S$ be a subpath of $P$ of length $\ell(\delta)$. Denote by $L$ (resp., $R$ ) the subpath of 
$P$ to the "left" (resp., "right") of $S$. Informally, if the length of $S$ is larger than $2 t+1$, then $S$ serves as a separator between the two subpaths $L$ and $R$. This follows since as algorithm $\mathcal{A}$ runs in $t$ rounds, each node in $P$ is affected only by its $t$ neighborhood. As the $t$ neighborhood of every node $u \in L$ and $v \in R$ do not intersect, the events $\mathcal{E}(P, \mathbf{x}, \mathrm{Id}, L)$ and $\mathcal{E}(P, \mathbf{x}, \mathrm{Id}, R)$ are independent.

The secureness property becomes useful when bounding the probability that at least some node in $P$ says "no". A natural approach to upper bound this probability is by applying a union bound on the events $\mathcal{E}(P, \mathbf{x}, \mathrm{Id}, V(L) \cup V(R))$ and $\mathcal{E}(P, \mathbf{x}, \mathrm{Id}, V(S))$. Letting $\mathcal{E}^{\prime}$ denote the event complementary to $\mathcal{E}(P, \mathbf{x}, \operatorname{Id}, V(P))$, we have

$$
\begin{aligned}
\operatorname{Pr}\left[\mathcal{E}^{\prime}\right]= & 1-\operatorname{Pr}[\mathcal{E}(P, \mathbf{x}, \mathrm{Id}, V(P))] \\
\leq & (1-\operatorname{Pr}[\mathcal{E}(P, \mathbf{x}, \mathrm{Id}, V(L))] \\
& \cdot \operatorname{Pr}[\mathcal{E}(P, \mathbf{x}, \mathrm{Id}, V(R))]) \\
& +(1-\operatorname{Pr}[\mathcal{E}(P, \mathbf{x}, \operatorname{Id}, V(S))]) \\
\leq \quad & 1-\operatorname{Pr}[\mathcal{E}(P, \mathbf{x}, \mathrm{Id}, V(L))] \\
& \cdot \operatorname{Pr}[\mathcal{E}(P, \mathbf{x}, \mathrm{Id}, V(R))]+\delta .
\end{aligned}
$$

The specific choice of $\lambda$ and $\delta$ depends on the context. Informally, the guiding principle is to set $\delta$ small enough so that the role of the central section $S$ can be neglected, while dealing separately with the two extreme sections $L$ and $R$ become manageable for they are sufficiently far apart.

\section{The $B_{k}$ hierarchy is strict}

In this section we show that the classes $B_{k}, k \geq 1$, form an infinite hierarchy of distinct classes, thereby proving that the general ability to boost the probability of success for a randomized decision problem is quite limited. In fact, we show separation in a very strong sense: there are decision problems in $B_{k+1}(0)$, i.e., that have a $(p, q)$-decider running in zero rounds with $p^{1+1 /(k+1)}+q>1$, which cannot be decided by a $(p, q)$-decider with $p^{1+1 / k}+q>1$, even if the number of rounds of the latter is as large as $n^{1-\varepsilon}$ for every fixed $\varepsilon>0$.

Theorem 3.1 $B_{k+1}(0) \backslash B_{k}(t) \neq \emptyset$ for every $k \geq 1$ and every $t=o(n)$.

Proof: Let $k$ be any positive integer. We consider the following distributed language, which is a generalized variant AMOS $-k$ of the problem AMOS introduced in [13]. As in AMOS, the input $\mathbf{x}$ of AMOS- $k$ satisfies $\mathbf{x} \in\{0,1\}^{n}$, i.e., each node $v$ is given as input a boolean $\mathbf{x}(v)$. The language AMOS- $k$ is then defined by:

$$
\begin{gathered}
\text { At-Most-k-Selected }(\text { AMOS }-k)= \\
\left\{(G, \mathbf{x}) \text { s.t. }\|\mathbf{x}\|_{1} \leq k\right\} .
\end{gathered}
$$

Namely, AMOS $-k$ consists of all instances containing at most $k$ selected nodes (i.e., at most $k$ nodes with input 1), with all other nodes unselected (having input 0). In order to prove Theorem 3.1, we show that AMOS $-k \in B_{k+1}(0) \backslash B_{k}(t)$ for every $t=o(n)$.

We first establish that AMOS $-k$ belongs to $B_{k+1}(0)$. We adapt algorithm $\mathcal{A}$ presented in [13] for AMOS to the case of AMOS $-k$. The following simple randomized algorithm runs in 0 time: every node $v$ which is not selected, i.e., such that $\mathbf{x}(v)=0$, says "yes" with probability 1 ; and every node which is selected, i.e., such that $\mathbf{x}(v)=1$, says "yes" with probability $p^{1 / k}$, and "no" with probability $1-p^{1 / k}$. If the graph has $s \leq k$ nodes selected, then all nodes say "yes" with probability $p^{s / k} \geq p$, as desired. On the other hand, if there are $s \geq k+1$ selected nodes, then at least one node says "no" with probability 
$1-p^{s / k} \geq 1-p^{(k+1) / k}=1-p^{1+1 / k}$. We therefore get a $(p, q)$-decider with $p^{1+1 / k}+q \geq 1$, that is, such that $p^{1+1 /(k+1)}+q>1$. Thus AMOS $-k \in B_{k+1}(0)$.

We now consider the harder direction, and prove that AMOS $-k \notin B_{k}(t)$, for any $t=o(n)$. To prove this separation, it is sufficient to consider AMOS $-k$ restricted to the family of $n$-node paths. Fix a function $t=o(n)$, and assume, towards contradiction, that there exists a distributed $(p, q)$-decider $\mathcal{A}$ for AMOS $-k$ that runs in $O(t)$ rounds, with $p^{1+1 / k}+q>1$. Let $\varepsilon \in(0,1)$ be such that $p^{1+1 / k+\varepsilon}+q>1$. Let $P$ be an $n$-node path, and let $S \subset P$ be a subpath of $P$. Let $\delta \in[0,1]$ be a constant satisfying

$$
0<\delta<p^{1+1 / k}\left(1-p^{\varepsilon}\right) / k .
$$

Consider a positive instance and a negative instance of AMOS $-k$, respectively denoted by

$$
I=(P, \mathbf{x}) \text { and } I^{\prime}=\left(P, \mathbf{x}^{\prime}\right) .
$$

Both instances are defined on the same $n$-node path $P$, where $n \geq k(\ell(\delta)+1)+1$.

Recall that $\ell(\delta)=\ell(\delta, 2 t+1)$ (see Eq. (1p). We consider executions of $\mathcal{A}$ on these two instances, where nodes are given the same id's. Both instances have almost the same input. In particular, the only difference is that instance $I$ contains $k$ selected nodes, whereas $I^{\prime}$ has the same selected nodes as $I$ plus one additional selected node. Therefore $I$ is legal, while $I^{\prime}$ is illegal. In $I^{\prime}$, the path $P$ is composed of $k+1$ sections, each containing a unique selected node, and where each pair of consecutive sections separated by a $\delta$-secure subpath. More precisely, let us enumerate the nodes of $P$ from 1 to $n$, with node $v$ adjacent to nodes $v-1$ and $v+1$, for every $1<v<n$. Consider the $k$ subpaths of $P$ defined by:

$$
S_{i}=[(i-1) \ell(\delta)+i+1, i \cdot \ell(\delta)+i]
$$

for $i=\{1, \ldots, k\}$. Let the selected nodes in $I^{\prime}$ be positioned as follows. Let $u_{1}=1$ and let $u_{i}=$ $(i-1) \ell(\delta)+i$ for $i=2, \ldots, k+1$. Then set

$$
\mathbf{x}^{\prime}(v)= \begin{cases}1, & \text { if } v=u_{i} \text { for some } i \in\{1, \ldots, k+1\} \\ 0, & \text { otherwise. }\end{cases}
$$

See Fig. 2(a) for a schematic representation of $I^{\prime}$. Our next goal is to define the legal instance $I=(P, \mathbf{x})$. To do so, we begin by claiming that each $S_{i}$ contains a $\delta$-secure internal subpath $S_{i}^{\prime}=\left[a_{i}, b_{i}\right]$. Naturally, we would like to employ Fact 2.2. However, Fact 2.2 refers to subpaths of valid instances $(P, \mathbf{x}) \in \mathcal{L}$, and $I^{\prime}$ is illegal. So instead, let us focus on the instance $\left(S_{i}, \mathbf{x}_{S_{i}}^{\prime}\right)$. Since $\left(S_{i}, \mathbf{x}_{S_{i}}^{\prime}\right)$ contains no leaders, $\left\|\mathbf{x}_{S_{i}}^{\prime}\right\|_{1}=0$, it follows that $\left(S_{i}, \mathbf{x}_{S_{i}}^{\prime}\right) \in \mathcal{L}$, and Fact 2.2 can be applied on it. Subsequently, since $\left|S_{i}\right|>\ell(\delta)$ it follows that $S_{i}$ contains an internal $\delta$-secure subpath $S_{i}^{\prime}=\left[a_{i}, b_{i}\right]$, whose $t$ neighborhood is strictly in $S_{i}$. Therefore, when applying algorithm $\mathcal{A}$ on $\left(S_{i}, \mathbf{x}_{S_{i}}^{\prime}, \operatorname{Id}_{S_{i}}\right)$ and on $\left(P, \mathbf{x}^{\prime}, \mathrm{Id}\right)$, the nodes in the $(2 t+1)$-length segment $S_{i}^{\prime}$ behave the same, thus $\operatorname{Pr}\left[\mathcal{E}\left(P, \mathbf{x}^{\prime}, \operatorname{Id}, V\left(S_{i}^{\prime}\right)\right)\right]=\operatorname{Pr}\left[\mathcal{E}\left(S_{i}, \mathbf{x}_{S_{i}}^{\prime}, \operatorname{Id}_{S_{i}}, V\left(S_{i}^{\prime}\right)\right)\right]$. Hence, $S_{i}^{\prime}$ is a $\delta$-secure subpath in $\left(P, \mathbf{x}^{\prime}\right.$, Id $)$ as well, for every $i \in\{1, \ldots, k\}$, see Fig. 2(b).

The $\delta$-secure subpaths $S_{i}^{\prime}$ 's are now used to divide $P$ into $2 k+1$ segments. Specifically, there are $k+1$ segments $T_{i}, i=1, \ldots, k+1$, each with one selected node. The $\delta$-secure subpaths $S_{i}^{\prime}=\left[a_{i}, b_{i}\right]$ separate $T_{i}$ from $T_{i+1}$. More precisely, set $T_{1}=\left[1, a_{1}-1\right], T_{i}=\left[b_{i-1}+1, a_{i}-1\right]$ for $i \in 2, \ldots, k$, and $T_{k+1}=\left[b_{k}+1, n\right]$, getting

$$
P=T_{1} \circ S_{1}^{\prime} \circ T_{2} \circ S_{2}^{\prime} \circ \ldots \circ T_{k} \circ S_{k}^{\prime} \circ T_{k+1}
$$

where $\circ$ denotes path concatenation. Let $\mathcal{T}_{i}=\mathcal{E}\left(P, \mathbf{x}^{\prime}, \mathrm{Id}, V\left(T_{i}\right)\right)$ be the event that all nodes in the subpath $T_{i}$ say "yes" in the instance $I^{\prime}$, for $i \in\{1, \ldots, k+1\}$ and let $p_{i}=\operatorname{Pr}\left[\mathcal{T}_{i}\right]$ be its probability. Let $j$ be such that $p_{j}=\max _{i} p_{i}$. We are now ready to define the valid instance $I=(P, \mathbf{x})$ :

$$
\mathbf{x}(v)= \begin{cases}1, & \text { if } v=u_{i} \text { for some } i \in\{1, \ldots, k+1\} \\ & i \neq j \\ 0, & \text { otherwise }\end{cases}
$$


Note that $\left\|\mathbf{x}^{\prime}\right\|_{1}=k+1$ and $\|\mathbf{x}\|_{1}=k$, thus $I \in$ AMOS $-k$ while $I^{\prime} \notin$ AMOS $-k$. See Fig. 2(c,d) for an illustration of $I$ versus $I^{\prime}$.

We now make the following observation.

Claim 3.2 $\forall i \neq j, \operatorname{Pr}\left[\mathcal{E}\left(P, \mathbf{x}, \mathrm{Id}, V\left(T_{i}\right)\right)\right]=p_{i}$.

Proof: This follows since the distance between any two nodes $u$ (resp., $v$ ) in distinct $T_{i}^{\prime} s$ is greater than $t$, which implies that $\mathbf{x}\left(L_{i} \circ T_{i} \circ R_{i}\right)=\mathbf{x}^{\prime}\left(L_{i} \circ T_{i} \circ R_{i}\right)$ where $L_{i}$ (resp., $\left.R_{i}\right)$ is the subpath of length $t$ to the left (resp., to the right) of $T_{i}$ in $P$, from which it follows that under $\mathcal{A}$ the nodes of $T_{i}$ have the same behavior in both instances $I$ and $I^{\prime}$.

Let $\mathcal{N}$ (resp., $\mathcal{N}^{\prime}$ ) be the event that there exists at least one node in $I$ (resp., $I^{\prime}$ ) that says "no" when applying algorithm $\mathcal{A}$. Similarly, let $\mathcal{Y}$ (resp., $\mathcal{Y}^{\prime}$ ) be the event stating that all nodes in the configuration $I$ (resp., $I^{\prime}$ ) say "yes". Let $\mathcal{T}=\bigcup_{i=1}^{k+1} \mathcal{T}_{i}$ be the event that all nodes in each subpaths $T_{i}$, for $i \in\{1, \ldots, k+1\}$ say "yes" in the instance $I^{\prime}$. For every $i \in\{1, \ldots, k\}$, let $\mathcal{S}_{i}=\mathcal{E}\left(P, \mathbf{x}^{\prime}, \operatorname{Id}, V\left(S_{i}^{\prime}\right)\right)$ be the event that all nodes in the $\delta$-secure subpath $S_{i}^{\prime}$ say "yes" in the instance $I^{\prime}$. We have $\operatorname{Pr}(\mathcal{Y})=\operatorname{Pr}[\mathcal{E}(P, \mathbf{x}, \operatorname{Id}, V(P))]$ and $\operatorname{Pr}\left(\mathcal{Y}^{\prime}\right)=$ $\operatorname{Pr}\left[\mathcal{E}\left(P, \mathbf{x}^{\prime}\right.\right.$, Id,$\left.V(P)\right)$, while $\operatorname{Pr}(\mathcal{N})=1-\operatorname{Pr}(\mathcal{Y})$ and $\operatorname{Pr}\left(\mathcal{N}^{\prime}\right)=1-\operatorname{Pr}\left(\mathcal{Y}^{\prime}\right)$.

Since $\mathcal{A}$ is a $(p, q)$-decider, as we assume by contradiction that AMOS-k in $B_{k}$, we have $\operatorname{Pr}\left(\mathcal{N}^{\prime}\right) \geq q$, and thus $\operatorname{Pr}\left(\mathcal{N}^{\prime}\right)>1-p^{1+1 / k+\varepsilon}$. Therefore, $\operatorname{Pr}\left(\mathcal{Y}^{\prime}\right)<p^{1+1 / k+\varepsilon}$. Moreover, since $I \in$ AMOS $-k$, we also have that $\operatorname{Pr}(\mathcal{Y}) \geq p$. Therefore, the ratio $\hat{\rho}=\operatorname{Pr}\left(\mathcal{Y}^{\prime}\right) / \operatorname{Pr}(\mathcal{Y})$ satisfies

$$
\hat{\rho}<p^{1 / k+\varepsilon} .
$$

On the other hand, note that by applying the union bound to the $k+1$ events $\mathcal{T}, \bigcup_{i=1}^{k} \mathcal{S}_{i}$, we get

$$
\begin{aligned}
\operatorname{Pr}\left(\mathcal{N}^{\prime}\right) & \leq(1-\operatorname{Pr}[\mathcal{T}])+\left(\sum_{i=1}^{k}\left(1-\operatorname{Pr}\left[\mathcal{S}_{i}\right]\right)\right) \\
& \leq 1-p_{j} \cdot \prod_{i \neq j} p_{i}+k \cdot \delta
\end{aligned}
$$

where the last inequality follows by the fact that each $S_{i}^{\prime}$ is a $(\delta, 2 t+1)$-secure subpath, thus the events $\mathcal{T}_{i_{1}}, \mathcal{T}_{i_{2}}$ are independent for every $i_{1}, i_{2} \in\{1, \ldots, k+1\}$ (since the distance between any two nodes $u \in T_{i_{1}}$ and $v \in T_{i_{2}}$ is at least $\left.2 t+1\right)$. This implies that $\operatorname{Pr}\left(\mathcal{Y}^{\prime}\right) \geq p_{j} \cdot \prod_{i \neq j} p_{i}-k \cdot \delta$. Since $\operatorname{Pr}(\mathcal{Y}) \leq \prod_{i \neq j} p_{i}$ (by the independence of the events $\mathcal{T}_{i_{1}}, \mathcal{T}_{i_{2}}$, for every $i_{1}, i_{2} \in\{1, \ldots k+1\}$ ), it then follows that the ratio $\hat{\rho}$ satisfies

$$
\begin{aligned}
\hat{\rho} & \geq \frac{p_{j} \cdot \prod_{i \neq j} p_{i}-k \cdot \delta}{\prod_{i \neq j} p_{i}} \\
& \geq p_{j}-\frac{k \cdot \delta}{\prod_{i \neq j} p_{i}} \geq p_{j}-k \cdot \delta / p,
\end{aligned}
$$

where the last inequality follows by the fact that $I \in \mathrm{AMOS}-k$ and thus $\prod_{i \neq j} p_{i} \geq \operatorname{Pr}(\mathcal{Y}) \geq p$. Finally, note that $p_{j} \geq p^{1 / k}$. This follows since $p_{j} \geq p_{i}$ for every $i \in\{1, \ldots, k+1\}$, so $p_{j}^{k} \geq \prod_{i \neq j} p_{i} \geq p$. By Eq. (4), we then have that $\hat{\rho} \geq p^{1 / k}-k \cdot \delta / p$. Combining this with Eq. 3 , we get that $p^{1 / k}-k \cdot \delta / p<p^{1 / k+\varepsilon}$, which is in contradiction to the definition of $\delta$ in Eq. 2 .

Finally, we show that the $B_{k}(t)$ hierarchy does not capture all languages even for $k=\infty$ and $t$ as large as $o(n)$. The proof of the following theorem is deferred to the Appendix.

Theorem 3.3 There is a language not in $B_{\infty}(t)$, for every $t=o(n)$. 


\section{A sharp threshold between determinism and randomization}

It is known [13] that beyond the threshold $p^{2}+q=1$, randomization does not help. This result however holds only for a particular type of languages, called hereditary, i.e., closed under inclusion. In this section, we provide one more evidence supporting our belief that the threshold $p^{2}+q=1$ identified in [13] holds for all languages, and not only for hereditary languages. Indeed, we prove that, restricted to path topologies and finite inputs, every language $\mathcal{L}$ for which there exists a $(p, q)$-decider running in constant time, with $p^{2}+q>1$, can actually be decided deterministically in constant time.

Theorem 4.1 Let $\mathcal{L}$ be a distributed language restricted to paths, with a finite set of input values. If $\mathcal{L} \in$ $B_{1}(O(1))$, then $\mathcal{L} \in \operatorname{LD}(O(1))$.

Proof: Let $\mathcal{L} \in B_{1}(O(1))$ be a distributed language restricted to paths, and defined on the (finite) input set $\Sigma$. Consider a distributed $(p, q)$-decider $\mathcal{A}$ for $\mathcal{L}$ that runs in $t=O(1)$ rounds, with $p^{2}+q>1$. Fix a constant $\delta$ such that $0<\delta<p^{2}+q-1$.

Given a subpath $S$ of a path $P$, let us denote by $S_{l}$ (respectively, $S_{r}$ ) the subpath of $P$ to the left (resp., right) of $S$, so that $P=S_{l} \circ S \circ S_{r}$.

Informally, a collection of three paths $P, P^{\prime}$, and $P^{\prime \prime}$ (of possibly different lengths) is called a $\lambda$-path triplet if (1) the inputs of those paths agree on some "middle" subpath of size at least $\lambda$, (2) paths $P$ and $P^{\prime \prime}$ coincide on their corresponding "left" parts, and (3) paths $P^{\prime}$ and $P^{\prime \prime}$ coincide on their "right" parts. See Figure 4. Formally, a $\lambda$-path triplet is a triplet $\left[(P, S, \mathbf{x}),\left(P^{\prime}, S^{\prime}, \mathbf{x}^{\prime}\right),\left(P^{\prime \prime}, S^{\prime \prime}, \mathbf{x}^{\prime \prime}\right)\right]$ such that $|P|,\left|P^{\prime}\right|,\left|P^{\prime \prime}\right| \geq \lambda$, $\mathbf{x}, \mathbf{x}^{\prime}, \mathbf{x}^{\prime \prime}$ are inputs on these paths, respectively, and $S \subset P, S^{\prime} \subset P^{\prime}, S^{\prime \prime} \subset P^{\prime \prime}$ are three subpaths satisfying (1) $|S|=\left|S^{\prime}\right|=\left|S^{\prime \prime}\right| \geq \lambda$, (2) $\mathbf{x}_{S}=\mathbf{x}_{S^{\prime}}^{\prime}=\mathbf{x}_{S^{\prime \prime}}^{\prime \prime}$, and (3) $\mathbf{x}_{S_{l}^{\prime \prime}}^{\prime \prime}=\mathbf{x}_{S_{l}}$ and $\mathbf{x}_{S_{r}^{\prime \prime}}^{\prime \prime}=\mathbf{x}_{S_{r}^{\prime}}^{\prime}$. The proof of the following claim is deferred to the Appendix.

Claim 4.2 Let $\left[(P, S, \mathbf{x}),\left(P^{\prime}, S^{\prime}, \mathbf{x}^{\prime}\right),\left(P^{\prime \prime}, S^{\prime \prime}, \mathbf{x}^{\prime \prime}\right)\right]$ be a $\lambda$-path triplet. If $\lambda \geq \ell(\delta)$, for $\ell$ as defined in Eq. $\left\{1\right.$, then $\left((P, \mathbf{x}) \in \mathcal{L}\right.$ and $\left.\left(P^{\prime}, \mathbf{x}^{\prime}\right) \in \mathcal{L}\right) \Rightarrow\left(P^{\prime \prime}, \mathbf{x}^{\prime \prime}\right) \in \mathcal{L}$.

We now observe that, without loss of generality, one can assume that in all instances $(P, \mathbf{x})$ of $\mathcal{L}$, the two extreme vertices of the path $P$ have a special input symbol $\otimes$. To see why this holds, let $\otimes$ be a symbol not in $\Sigma$, and consider the following language $\mathcal{L}^{\prime}$ defined over $\Sigma \cup\{\otimes\}$. Language $\mathcal{L}^{\prime}$ consists of instances $(P, \mathbf{x})$ such that (1) the endpoints of $P$ have input $\otimes$, and (2) $\left(P^{\prime}, \mathbf{x}^{\prime}\right) \in \mathcal{L}$, where $P^{\prime}$ is the path resulting from removing the endpoints of $P$, and where $\mathbf{x}_{v}^{\prime}=\mathbf{x}_{v}$ for every node $v$ of $P^{\prime}$. Any $(p, q)$ decider algorithm for $\mathcal{L}$ (resp., $\mathcal{L}^{\prime}$ ), can be trivially transformed into a $(p, q)$ decider algorithm for $\mathcal{L}^{\prime}$ (resp., $\mathcal{L}$ ) with the same success guarantees and running time. Hence, in the remaining of the proof, we assume that in all instances $(P, \mathbf{x}) \in \mathcal{L}$, the two extreme vertices of the path $P$ have input $\otimes$.

We say that a given instance $(P, \mathbf{x})$ is extendable if there exists an extension of it in $\mathcal{L}$, i.e., if there exists an instance $\left(P^{\prime}, \mathbf{x}^{\prime}\right) \in \mathcal{L}$ such that $P \subseteq P^{\prime}$ and $\mathbf{x}_{P}^{\prime}=\mathbf{x}$. The proof of the following claim is deferred to the Appendix.

Claim 4.3 There exists a (centralized) algorithm $\mathcal{X}$ that, given any configuration $(P, \mathbf{x})$ with $|P| \leq 2 \ell(\delta)+$ 1 , decides whether $(P, \mathbf{x})$ is extendable. Moreover, algorithm $\mathcal{X}$ uses messages of constant size.

We may assume, hereafter, that such an algorithm $\mathcal{X}$, as promised by Claim 4.3 , is part of the language specification given to the nodes. We show that $\mathcal{L} \in \mathrm{LD}(O(t))$ by proving the existence of a deterministic algorithm $\mathcal{D}$ that recognizes $\mathcal{L}$ in $O(t)$ rounds. Given a path $P$, an input $\mathbf{x}$ over $P$, and an identity assignment Id, algorithm $\mathcal{D}$ applied at a node $u$ of $P$ operates as follows. If $\mathbf{x}_{u}=\otimes$ then $u$ outputs "yes" if and only if $u$ is an endpoint of $P$. Otherwise, i.e., if $\mathbf{x}_{u} \neq\{\otimes\}$, then $u$ outputs "yes" if and only if $\left(B_{u}, \mathbf{x}_{B_{u}}\right)$ is extendable (using algorithm $\mathcal{X}$ ), where $B_{u}=B(u, \ell(\delta)$ ) is the ball centered at $u$, and of radius $\ell(\delta)$ in $P$. 
Algorithm $\mathcal{D}$ is a deterministic algorithm that runs in $\ell(\delta)$ rounds. We claim that Algorithm $\mathcal{D}$ recognizes $\mathcal{L}$. To establish that claim, consider first an instance $(P, \mathbf{x}) \in \mathcal{L}$. For every node $u,(P, \mathbf{x}) \in \mathcal{L}$ is an extension of $\left(B_{u}, \mathbf{x}_{B_{u}}\right)$. Therefore, every node $u$ outputs "yes", as desired. Now consider an instance $(P, \mathbf{x}) \notin \mathcal{L}$. Assume, for the purpose of contradiction, that there exists an identity assignment Id such that, when applying $\mathcal{D}$ on $(P, \mathbf{x}, \mathrm{Id})$, every node $u$ outputs "yes".

Claim 4.4 In this case, $|P|>2 \ell(\delta)+1$.

Proof: Assume by contradiction that $|P| \leq 2 \ell(\delta)+1$, and consider the middle node $s$ of $P$. Since $s$ outputs "yes", it follows that $(P, \mathbf{x})$ can be extended to $\left(P^{\prime}, \mathbf{x}^{\prime}\right)$ such that $\left(P^{\prime}, \mathbf{x}^{\prime}\right) \in \mathcal{L}$. However, since the extremities of $P$ output "yes", it means that their input is $\otimes$. Therefore, as $\left|P^{\prime}\right|>|P|$, we get that there is an internal node of $P^{\prime}$ which has input $\otimes$, contradicting $\left(P^{\prime}, \mathbf{x}^{\prime}\right) \in \mathcal{L}$.

Let $S \subseteq P$ be the longest subpath of $P$ such that there exists an extension $\left(P^{\prime}, \mathbf{x}^{\prime}\right)$ of $\left(S, \mathbf{x}_{S}\right)$, with $\left(P^{\prime}, \mathbf{x}^{\prime}\right) \in \mathcal{L}$. Since $|P|>2 \ell(\delta)+1$, and since the middle node of $P$ outputs "yes", we have $|S| \geq 2 \ell(\delta)+1$. The proof carries on by distinguishing two cases for the length of $S$.

If $S=P$, then $(P, \mathbf{x})$ can be extended to $\left(P^{\prime}, \mathbf{x}^{\prime}\right) \in \mathcal{L}$. By the same arguments as above, since each extremity $w$ of $P$ has input $\otimes$, we conclude that $P=P^{\prime}$, with $\mathbf{x}=\mathbf{x}^{\prime}$. Contradicting the fact that $(P, \mathbf{x}) \notin \mathcal{L}$. Therefore $2 \ell(\delta)+1 \leq|S|<|P|$. Let $a$ and $b$ be such that $S=[a, b]$. As $S$ is shorter than $P$, it is impossible for both $a$ and $b$ to be endpoints of $P$. Without loss of generality, assume that $a$ is not an endpoint of $P$. Since $a$ outputs "yes", there exists an extension $\left(P^{\prime \prime}, \mathbf{x}^{\prime \prime}\right) \in \mathcal{L}$ of $\left(B_{a}, \mathbf{x}_{B_{a}}\right)$. In fact, $\left(P^{\prime \prime}, \mathbf{x}^{\prime \prime}\right)$ is also an extension of $\mathbf{x}_{[a, a+\ell(\delta)]}$. Since $\mathbf{x}^{\prime}$ and $\mathbf{x}^{\prime \prime}$ agree on $[a, a+\ell(\delta)]$, and since both $\left(P^{\prime}, \mathbf{x}^{\prime}\right)$, and $\left(P^{\prime \prime}, \mathbf{x}^{\prime \prime}\right)$ are in $\mathcal{L}$, we get from Lemma 4.2 that $\mathbf{x}_{[a-1, b]}$ can be extended to an input $\left(P^{\prime \prime \prime}, \mathbf{x}^{\prime \prime \prime}\right) \in \mathcal{L}$, which contradicts the choice of $S$. The theorem follows.

\section{On the impossibility of boosting}

Theorems 3.1 and 3.3 demonstrate that boosting the probability of success might be doable, but only from $(p, q)$ satisfying $p^{1+1}(k+1)+q>1$ to $(p, q)$ satisfying $p^{1+1 / k}+q>1$ (with the extremes excluded). In this section, we prove that once the inputs may be restricted in certain ways, the ability to boost the success probability become almost null. More precisely, recall that so far we considered languages as collections of pairs $(G, \mathbf{x})$ where $G$ is a (connected) $n$-node graph and $\mathbf{x} \in \Sigma^{n}$ is the input vector to the nodes of $G$, in some finite of infinite alphabet $\Sigma$, that is, $\mathbf{x}(v) \in \Sigma$ for all $v \in V(G)$. An instance of an algorithm $\mathcal{A}$ deciding a language $\mathcal{L}$ was defined as any such pair $(G, \mathbf{x})$. We now consider the case where the set of instances is restricted to some specific subset of inputs $\mathcal{I} \subset \Sigma^{n}$. That is, the distributed algorithm $\mathcal{A}$ has now the promise that in the instances $(G, \mathbf{x})$ admissible as inputs, the input vector $\mathbf{x}$ is restricted to $\mathbf{x} \in \mathcal{I} \subset \Sigma^{n}$.

We define the classes $C_{r}(t)$ in a way identical to the classes $B_{k}(t)$, but generalized in two ways. First, the parameter $r$ is not bounded to be integral, but can be any positive real. Second, the decision problems under consideration are extended to the ones in which the set of input vectors $\mathbf{x}$ can be restricted. So, in particular, $B_{k}(t) \subseteq C_{k}(t)$, for every positive integer $k$, and every function $t$. The following theorem proves that boosting can made as limited as desired. The proof of this theorem is deferred to the Appendix.

Theorem 5.1 Let $r<r^{\prime}$ be any two positive reals. Then, $C_{r^{\prime}}(0) \backslash C_{r}(t) \neq \emptyset$ for every $t=o(n)$.

Note that Theorem 5.1 demonstrates not only the (almost) inability of boosting the probability of success when the inputs to the nodes are restricted to specific kinds, but also the inability of derandomizing, even above the threshold $p^{2}+q=1$. Indeed, the following is a direct consequence of Theorem 5.1

Corollary 5.2 For every positive real $r$, there is a decision problem in $C_{r}(0)$ which cannot be decided deterministically in o $n$ ) rounds. 


\section{APPENDIX}

\section{A Some proofs}

Proof of Fact 2.2; Fix any identity assignment Id and let $S \subset P$ be a subpath of length $d \geq \ell(\delta, \lambda)$, where $S=\left(v_{1}, \ldots, v_{d}\right)$. In what follows, we may override the nodes $v_{i}$ with their indices in $S$, namely $i$. Let $r_{\ell}=\lceil(\lambda-1) / 2\rceil$. Given a vertex $v_{i} \in S$, for $i \in\left(r_{\ell}, d-r_{\ell}\right)$, let $B_{i}$ be the subpath of its $r_{\ell}$ neighborhood in $S$. Formally, $B_{i}=\left[i-r_{\ell}, i+r_{\ell}\right]$. In addition, let $\mathcal{B}_{i}$ be the event that all nodes in $B_{i}$ say "yes" when applying $\mathcal{A}$ on $P$. That is $\mathcal{B}_{i}=\mathcal{E}\left(P, \mathbf{x}, \operatorname{Id}, V\left(B_{i}\right)\right)$. Let

$$
R=\left(r_{\ell}+t+1, d-r_{\ell}-t\right)
$$

be the ranges of indices $i$ whose $r_{\ell}$ neighborhood $B_{i}$ is internal in $S$, i.e., $B_{i} \subset[t+1, d-t-1]$. That is by definition, for every $i \in R$, it holds that $B_{i}$ is at least of length $\lambda$ and is internal in $S$. See Fig. 1 for illustration. We would like to show that there exists $i^{*} \in R$, such that $B_{i^{*}}$ is a $(\delta, \lambda)$-secure subpath, i.e., that $\operatorname{Pr}\left[\mathcal{B}_{i}\right] \geq 1-\delta$.

To establish the existence of $i^{*} \in R$, we bound from above the size of the set $\mathcal{H}$ containing all indices $j \in R$ whose $B_{j}$ is not a $(\delta, \lambda)$-secure subpath. Formally, define

$$
\mathcal{H}=\left\{j \in R \mid \operatorname{Pr}\left[\mathcal{B}_{j}\right]<1-\delta\right\} .
$$

We next upper bound the size of $\mathcal{H}$, by covering the integers in $R$ by at most

$$
Q=2\left(t+r_{\ell}\right)+1
$$

sets, each being a $Q$-independent set. That is, every two integers in the same set, are at least $Q$ apart in $P$. Let $\mathcal{J}_{1}, \ldots, \mathcal{J}_{Q}$ be the $Q$-independent sets that cover the indices of $R$.

Specifically, for $s \in[1, Q]$ and $m(S)=\left\lceil\left(d-4\left(r_{\ell}+t\right)\right) / Q\right\rceil$, we define

$$
\mathcal{J}_{s}=\left\{s+r_{\ell}+t+1+j \cdot Q \mid j \in[0, m(S)]\right\} \cap[1, n] .
$$

Observe, that as desired, $R \subset \bigcup_{s \in[1, Q]} \mathcal{J}_{s}$, and for each $s \in[1, Q], \mathcal{J}_{s}$ is a $Q$-independent set. In what follows, fix $s \in[1, Q]$ and let $\mathcal{J}=\mathcal{J}_{s}$. Let $\mathcal{J}^{\prime}=\mathcal{J} \cap \mathcal{H}$, the indices in $\mathcal{J}$ whose $r_{\ell}$ neighborhood does not correspond to $(\delta, \lambda)$-secure subpath. On the one hand, since to a $(P, \mathbf{x}) \in \mathcal{L}$, we have that

$$
\operatorname{Pr}\left[\bigcup_{i \in \mathcal{J}^{\prime}} \mathcal{B}_{i}\right] \geq p
$$

On the other hand, observe that for every two indices $i_{1}, i_{2} \in \mathcal{J}^{\prime}$, it holds that the distance between every $u \in B_{i_{1}}$ and $v \in B_{i_{2}}$ is at least $2 t+1$ (since $J^{\prime}$ is $Q$-independent). Hence, the events $\mathcal{B}_{i_{1}}$ and $\mathcal{B}_{i_{2}}$ are independent. We therefore get that

$$
\operatorname{Pr}\left[\bigcup_{i \in \mathcal{J}^{\prime}} \mathcal{B}_{i}\right]=\prod_{i \in \mathcal{J}^{\prime}} \operatorname{Pr}\left[\mathcal{B}_{i}\right]<(1-\delta)^{\left|\mathcal{J}^{\prime}\right|},
$$

where the last inequality follows by the fact that $\mathcal{J}^{\prime} \subset \mathcal{H}$. Combining Eq. A.1 and A.2 we get that

$$
\left|\mathcal{J}^{\prime}\right|<\log p / \log (1-\delta)
$$


We are now ready to upper bound the size of $\mathcal{H}$. Noting that $S$ can be covered by an union of $Q$ sets $\mathcal{J}_{s}$ each of which is $Q$-independent, we get that

$$
|\mathcal{H}|=\sum_{i=1}^{Q}\left|\mathcal{J}_{i}^{\prime}\right|<Q \cdot \frac{\log p}{\log (1-\delta)},
$$

where $\mathcal{J}_{i}^{\prime}=\mathcal{J}_{i} \cap \mathcal{H}$. Finally note that $|R|>|\mathcal{H}|$. This follows by the fact that $|R|=|S|-2\left(r_{\ell}+t+1\right)$ and $|S| \geq \ell(\delta, \lambda)$. Hence,

$$
\begin{aligned}
|R| & =|S|-2\left(r_{\ell}+t+1\right) \\
& \geq \ell(\delta, \lambda)-2\left(r_{\ell}+t+1\right) \\
& \geq Q \cdot \frac{\log p}{\log (1-\delta)} \\
& >|\mathcal{H}|,
\end{aligned}
$$

where the last inequality follows by Eq. A.3). By the pigeonhole principle we get that there exists $i^{*} \in R$ which is not in $\mathcal{H}$. Thus $B_{i^{*}}$ is a $(\delta, \lambda)$-secure subpath, as required. The fact follows.

Proof of Theorem 3.3: We exhibit one specific language not in $B_{\infty}(t)$, for every $t=o(n)$. This language consists of determining whether the underlying network is acyclic. Specifically, let

$$
\text { Tree }=\{(G, \epsilon) \mid G \text { is a tree }\}
$$

where $\epsilon$ is the null input. Fix a function $t=o(n)$. Assume, towards contradiction, that there exists some finite $k$ such that Tree $\in B_{k}(t)$. Then there is a $(p, q)$-decider for Tree, given by $\mathcal{A}$, running in $t$ rounds, with $p^{1+1 / k}+q-1>0$. Hence, in particular, there exists some $\varepsilon>0$ such that $p+q-1>\varepsilon$. Define

$$
\delta=\varepsilon<p+q-1
$$

We consider graphs $G$ of size $n>\left\lceil\frac{21 \cdot \log p}{\log (1-\delta)}\right\rceil$. We will show that $\operatorname{Tree} \notin B_{k}(t)$ for any

$$
t \leq\left\lfloor\frac{\log (1-\delta)}{21 \cdot \log p}\right\rfloor \cdot n=o(n) .
$$

Consider the cycle $C$ with $n$ nodes labeled consecutively from 1 to $n$, and the path $P$ with nodes labeled consecutively from 1 to $n$. This labeling defines the identity assignment $\operatorname{Id}^{1}$. In the input configuration $(P, \epsilon)$, the probability that all nodes say "yes" when executing $\mathcal{A}$ is at least $p$. Let us identify a subpath $S=[x-t, \ldots, x+t+1]$ of $P$ to be used as an internal $(\delta, 2(t+1))$-secure subpath in $P$. I.e.,

$$
\operatorname{Pr}\left[\mathcal{E}\left(P, \epsilon, \operatorname{Id}^{1}, V(S)\right)\right] \geq 1-\delta .
$$

Note that, by Eq. 11, A.4, A.5), it follows that $n>\ell(\delta, 2(t+1))$. Hence by Fact 2.2, since $(P, \epsilon) \in \mathcal{L}$, there exists such internal subpath $S \subset P$. Consider the event $\mathcal{E}(P, \mathbf{x}, \mathrm{Id}, V(S))$ stating that all nodes in subpath $S$ of $P$ with input $\mathbf{x}$ and identity-assignment Id return "yes". We have that

$$
\operatorname{Pr}\left[\mathcal{E}\left(P, \epsilon, \operatorname{Id}^{1}, V(S)\right)\right]=\operatorname{Pr}\left[\mathcal{E}\left(C, \epsilon, \operatorname{Id}^{1}, V(S)\right)\right] .
$$

Consider a subpath $\widehat{S}$ composed of the subpath $S$ padded with a block $L$ of $t$ nodes before it and a block $R$ of $t$ nodes after it. Indeed, since $S$ is an internal subpath of $P$ (i.e., it is at distance at least $t+1$ from $P$ 's endpoints), the set of nodes of $\widehat{S}=L \circ S \circ R=[x-2 t, \ldots, x+2 t+1]$ appears consecutively in both $P$ 
and in $C$ with identity assignment $\mathrm{Id}^{1}$, and $\widehat{S}$ have the same identities (with $\mathrm{Id}^{1}$ ) and degrees in both $C$ and $P$. We now consider another identity-assignment $\operatorname{Id}^{2}$ for $P$, with nodes labeled consecutively from $x+1$ to $n$, and then from 1 to $x$. Consider the $(n-2(t+1))$-node subpath

$$
S^{\prime}=[x+t+2, \ldots, n, 1, \ldots, x-t-1] .
$$

We have

$$
\operatorname{Pr}\left[\mathcal{E}\left(P, \epsilon, \mathrm{Id}^{2}, V\left(S^{\prime}\right)\right)\right]=\operatorname{Pr}\left[\mathcal{E}\left(C, \epsilon, \operatorname{Id}^{1}, V\left(S^{\prime}\right)\right)\right],
$$

Consider a subpath $\widehat{S}^{\prime}$ composed of the subpath $S^{\prime}$ padded with a block $L^{\prime}$ of $t$ nodes before it and a block $R^{\prime}$ of $t$ nodes after it, i.e., $\widehat{S}^{\prime}=L^{\prime} \circ S^{\prime} \circ R^{\prime}$. Indeed, the set of $\widehat{S}^{\prime}$ nodes appears consecutively in both $C$ and $P$ with identity assignment $\mathrm{Id}^{2}$, and $L^{\prime} \circ S^{\prime} \circ R^{\prime}$ have the same identities (with $\mathrm{Id}^{2}$ ) and degrees in both $C$ and $P$, where $L^{\prime}$ (resp., $R^{\prime}$ ) is the subpath composed of the $t$ nodes with identities $\mathrm{Id}^{2}$ immediately larger than $x+t+1$ (resp., smaller than $x-t$ ). Formally, we have that $\operatorname{Id}_{\widehat{S}^{\prime}}^{1}=\operatorname{Id}_{\widehat{S}^{\prime}}^{2}$. See Fig. 3 for illustration. Let $\mathcal{S}=\mathcal{E}\left(C, \epsilon, \mathrm{Id}^{1}, V(S)\right)$, (resp., $\mathcal{S}^{\prime}=\mathcal{E}\left(C, \epsilon, \mathrm{Id}^{1}, V\left(S^{\prime}\right)\right)$ ) be the event that all nodes of $S \subset C$ (resp., $S^{\prime} \subset C$ ) say "yes". We can now combine these previous results to derive a contradiction. Since $C \notin$ Tree, by applying the union bound on the events $\mathcal{S}$ and $\mathcal{S}^{\prime}$, and using Eq. A.7) and (A.8), we get that

$$
\begin{aligned}
q \leq & 1-\operatorname{Pr}\left[\mathcal{E}\left(C, \epsilon, \mathrm{Id}^{1}, V(C)\right)\right] \\
\leq & \left(1-\operatorname{Pr}\left[\mathcal{S}^{\prime}\right]\right)+(1-\operatorname{Pr}[\mathcal{S}]) \\
= & \left(1-\operatorname{Pr}\left[\mathcal{E}\left(P, \epsilon, \mathrm{Id}^{2}, V\left(S^{\prime}\right)\right)\right]\right) \\
& +\left(1-\operatorname{Pr}\left[\mathcal{E}\left(P, \epsilon, \mathrm{Id}^{1}, V(S)\right)\right]\right) \\
\leq & \left(1-\operatorname{Pr}\left[\mathcal{E}\left(P, \epsilon, \mathrm{Id}^{2}, V\left(S^{\prime}\right)\right)\right]\right)+\delta
\end{aligned}
$$

where the last inequality holds by Eq. (A.6). Therefore we get

$$
q \leq 1-p+\delta
$$

by noticing that $\operatorname{Pr}\left[\mathcal{E}\left(P, \epsilon, \mathrm{Id}^{2}, V\left(S^{\prime}\right)\right)\right] \geq p$ since $P \in$ Tree. Finally, by Eq. A.4, we eventually get $q<1-p+p+q-1$ or $q<q$, contradiction.

Proof of Claim 4.2 Consider an identity assignment $\mathrm{Id}^{\prime \prime}$ for $\left(P^{\prime \prime}, \mathbf{x}^{\prime \prime}\right)$. Let $\mathrm{Id}$ and $\mathrm{Id}^{\prime}$ be identity assignments for $(P, \mathbf{x})$, and $\left(P^{\prime}, \mathbf{x}^{\prime}\right)$, respectively, which agree with $\mathrm{Id}^{\prime \prime}$ on the corresponding nodes. That is: (a) assignments $\mathrm{Id}, \mathrm{Id}^{\prime}$, and $\mathrm{Id}^{\prime \prime}$ agree on the nodes in $S, S^{\prime}$ and $S^{\prime \prime}$, respectively; (b) Id and $\mathrm{Id}^{\prime \prime}$ agree on the nodes in $S_{\ell}$ and $S_{\ell}^{\prime \prime}$, respectively; and (c) Id and $\mathrm{Id}^{\prime \prime}$ agree on the nodes in $S_{r}^{\prime}$ and $S_{r}^{\prime \prime}$, respectively. Since $(P, \mathbf{x}) \in \mathcal{L}$, and since $|S|=\lambda \geq \ell(\delta)$, it follows from Fact 2.2 that $S$ contains an internal $\delta$-secure subpath $H$. Then, let $H^{\prime}$ and $H^{\prime \prime}$ be the subpaths of $P^{\prime}$ and $P^{\prime \prime}$ corresponding to $H$. Since $S$ and $S^{\prime \prime}$ coincide in their inputs and identity assignments, then $H, H^{\prime}, H^{\prime \prime}$ have the same $t$-neighborhood in $P, P^{\prime}, P^{\prime \prime}$ respectively. Hence, $H^{\prime \prime}$ is also a $\delta$-secure (when running algorithm $\mathcal{A}$ in instance $\left.\left(P^{\prime \prime}, \mathbf{x}^{\prime \prime}\right)\right)$. Since both $(P, \mathbf{x})$ and $\left(P^{\prime}, \mathbf{x}^{\prime}\right)$ belong to $\mathcal{L}$, we have

$$
\left.\operatorname{Pr}\left[\mathcal{E}\left(H_{\ell}^{\prime \prime}, \operatorname{Id}^{\prime \prime}, \mathbf{x}^{\prime \prime}\right)=\operatorname{Pr}\left[\mathcal{E}\left(H_{\ell}, \operatorname{Id}, \mathbf{x}\right)\right]\right)\right] \geq p
$$

and

$$
\left.\operatorname{Pr}\left[\mathcal{E}\left(H_{r}^{\prime \prime}, \operatorname{Id}^{\prime \prime}, \mathbf{x}^{\prime \prime}\right)=\operatorname{Pr}\left[\mathcal{E}\left(H_{r}^{\prime}, \operatorname{Id}^{\prime}, \mathbf{x}^{\prime}\right)\right]\right)\right] \geq p .
$$

Moreover, as $\left|H^{\prime \prime}\right| \geq 2 t+1$, the two events $\mathcal{E}\left(H_{\ell}^{\prime \prime}, \mathrm{Id}^{\prime \prime}, \mathbf{x}^{\prime \prime}\right)$ and $\mathcal{E}\left(H_{r}^{\prime \prime}, \mathrm{Id}^{\prime \prime}, \mathbf{x}^{\prime \prime}\right)$ are independent. Hence

$$
\operatorname{Pr}\left[\mathcal{E}\left(H_{\ell}^{\prime \prime} \cup H_{r}^{\prime \prime}, \mathrm{Id}^{\prime \prime}, \mathbf{x}^{\prime \prime}\right)\right] \geq p^{2} .
$$


In other words, the probability that some node in $H_{\ell}^{\prime \prime} \cup H_{r}^{\prime \prime}$ says "no" is at most $1-p^{2}$. It follows, by union bound, that the probability that some node in $H^{\prime \prime}$ says "no" is at most $1-p^{2}+\delta<q$. Since $\mathcal{A}$ is a $(p, q)$-decider for $\mathcal{L}$, it cannot be the case that $\left(H^{\prime \prime}, \mathbf{x}^{\prime \prime}\right) \notin \mathcal{L}$.

Proof of Claim 4.3: Observe that since the running time $t$ is constant, then $2 \ell(\delta)+1$ is also constant. Therefore, there are only finitely many configurations $(\hat{P}, \mathbf{x})$ with $|\hat{P}| \leq 2 \ell(\delta)+1$ (since $\Sigma$ is finite). Call this set of configurations $\mathcal{C}$. Each of the configurations in $\mathcal{C}$ is either extendable or not. Hence, there exists a function $f: \mathcal{C} \rightarrow\{0,1\}$ such that for every configuration $C \in \mathcal{C}, f(C)=1$ if and only if $C$ is extendable. This function $f$ can be described in a finite manner, and hence gives rise to an algorithm as required by the claim.

Proof of Theorem 5.1; Let $\widehat{r}=a / b \in\left[r, r^{\prime}\right)$ be a positive rational where $a$ and $b$ are two co-prime integers. By the density of the rational numbers, such $\widehat{r}$ is guaranteed to exist. To establish the theorem, we consider the language AMOS- $a$ restricted to instances in $\mathcal{I}$, where

$$
\mathcal{I}=\left\{\mathbf{x} \in\{0,1\}^{*}:\|\mathbf{x}\|_{1} \notin[a+1, a+b-1]\right\} .
$$

In other words, the promise says that an input either satisfies AMOS $-a$, or is far from satisfying AMOS $-a$ (very many selected nodes). We prove that AMOS-a $\in C_{r^{\prime}}(0) \backslash C_{r}(t)$ for every $t=o(n)$.

We begin by showing that AMOS $-a \in C_{r^{\prime}}(0)$ by considering the following simple randomized algorithm that runs in 0 time: every node $v$ which is not selected, i.e., such that $\mathbf{x}(v)=0$, says "yes" with probability 1 ; and every node which is selected, i.e., such that $\mathbf{x}(v)=1$, says "yes" with probability $p^{1 / a}$, and "no" with probability $1-p^{1 / a}$. If the graph has $s \leq a$ nodes selected, then all nodes say "yes" with probability $p^{s / a} \geq p$, as desired. Else, there are $s \geq a+b$ leaders, (this follows from the promise), and at least one node says "no" with probability $1-p^{s / a} \geq 1-p^{(a+b) / a}=1-p^{1+1 / \widehat{r}}$. We therefore get a $(p, q)$-decider with $p^{1+1 / \widehat{r}}+q \geq 1$, thus $p^{1+1 / r^{\prime}}+q>1$ as $r^{\prime}>\widehat{r}$. It therefore follows that AMOS $-a \in C_{r^{\prime}}(0)$.

We now consider the harder direction, and prove that AMOS $-a \notin C_{r}(t)$, for any $t=o(n)$. Since $\widehat{r} \geq r$, it is sufficient to show that AMOS $-a \notin C_{\widehat{r}}(t)$. To prove this separation, consider the AMOS $-a$ problem restricted to the family of $n$-node paths. Fix a function $t=o(n)$, and assume, towards contradiction, that there exists a distributed $(p, q)$-decider $\mathcal{A}$ for AMOS $-a$ that runs in $O(t)$ rounds, with $p^{1+1 / \widehat{r}}+q>1$. Let $\varepsilon \in(0,1)$ be such that $p^{1+1 / \widehat{r}+\varepsilon}+q>1$. Let $P$ be an $n$-node path, and let $S \subset P$ be a subpath of $P$. Let $\delta \in[0,1]$ be a constant satisfying

$$
0<\delta<p^{1+1 / \widehat{r}}\left(1-p^{\varepsilon}\right) /(a+b-1) .
$$

Consider a positive instance and a negative instance of AMOS - a, respectively denoted by

$$
I=(P, \mathbf{x}) \text { and } I^{\prime}=\left(P, \mathbf{x}^{\prime}\right) .
$$

Both instances are defined on the same $n$-node path $P$, where

$$
n \geq(a+b-1)(\ell(\delta)+1)+1 .
$$

where $\ell(\delta)=\ell(\delta, 2 t+1)$, as defined by Eq. (1). We consider executions of $\mathcal{A}$ on these two instances, where nodes are given the same id's. Both instances have almost the same input. In particular, the only difference is that instance $I$ contains $a$ selected nodes, whereas $I^{\prime}$ has the same selected nodes as $I$ plus $b$ additional selected nodes. Therefore $I$ is legal, while $I^{\prime}$ is illegal. In addition, both inputs $\mathbf{x}$ and $\mathbf{x}^{\prime}$ satisfy the promise.

In $I^{\prime}$, the path $P$ is composed of $a+b$ sections, each containing a unique selected node, and where each pair of consecutive sections separated by $\delta$-secure subpaths. More precisely, let us enumerate the nodes of 
$P$ from 1 to $n$, with node $v$ adjacent to nodes $v-1$ and $v+1$, for every $1<v<n$. Consider the $a+b-1$ subpaths of $P$ defined by:

$$
S_{i}=[(i-1) \ell(\delta)+i+1, i \cdot \ell(\delta)+i]
$$

for $i=\{1, \ldots, a+b-1\}$. Let the selected nodes in $I^{\prime}$ be positioned as follows. Let $u_{1}=1$ and let $u_{i}=(i-1) \ell(\delta)+i$ for $i=2, \ldots, a+b$. Then set

$$
\mathbf{x}^{\prime}(v)= \begin{cases}1 & \text { if } v=u_{i} \text { for some } i \in\{1, \ldots, a+b\} \\ 0 & \text { otherwise }\end{cases}
$$

Our next goal is to construct a legal input $I=(P, \mathbf{x})$ with $a$ leaders. Towards this, we begin by showing that each $S_{i}$ contains a $\delta$-secure internal subpath $S_{i}^{\prime}=\left[x_{i}, y_{i}\right]$ (internal to $S_{i}$ ). Note that Fact 2.2 refers to subpaths in valid instances $(P, \mathbf{x}) \in \mathcal{L}$, and since $I^{\prime}$ is illegal it cannot be directly applied. So instead, let us focus on the instance $\left(S_{i}, \mathbf{x}_{S_{i}}^{\prime}\right)$ with IDs $\operatorname{Id}_{S_{i}}$. Since $S_{i}$ contains no leaders, $\left\|\mathbf{x}_{S_{i}}^{\prime}\right\|_{1}=0$, it follows that $\left(S_{i}, \mathbf{x}_{S_{i}}^{\prime}\right) \in \mathcal{L}$. Now we can safely apply Fact 2.2 . Indeed, since $\left|S_{i}\right|>\ell(\delta)$ it follows by the fact that $S_{i}$ contains an internal $\delta$-secure subpath $S_{i}^{\prime}=\left[x_{i}, y_{i}\right]$. Therefore, when applying algorithm $\mathcal{A}$ on $\left(S_{i}, \mathbf{x}_{S_{i}}^{\prime}, \operatorname{Id}_{S_{i}}\right)$ and on $\left(P, \mathbf{x}^{\prime}, \mathrm{Id}\right)$, the nodes of $S_{i}^{\prime}$ behave the same, thus $\operatorname{Pr}\left[\mathcal{E}\left(P, \mathbf{x}^{\prime}, \operatorname{Id}, V\left(S_{i}^{\prime}\right)\right)\right]=\operatorname{Pr}\left[\mathcal{E}\left(S_{i}, \mathbf{x}_{S_{i}}^{\prime}, \operatorname{Id}_{S_{i}}, V\left(S_{i}^{\prime}\right)\right)\right]$. Hence, $S_{i}^{\prime}$ is a $\delta$-secure subpath in $I^{\prime}$ as well, for every $i \in\{1, \ldots, a+b-1\}$.

The $\delta$-secure subpaths $S_{i}^{\prime}$ are used to divide $P$ into $2(a+b-1)+1$ segments. There are $a+b$ segments $T_{i}, i=1, \ldots, a+b$, each with one selected nodes. The $\delta$-secure subpaths $S_{i}^{\prime}=\left[x_{i}, y_{i}\right]$ separate $T_{i}$ from $T_{i+1}$. More precisely, we set

$$
T_{1}=\left[1, x_{1}-1\right], T_{i}=\left[y_{i-1}+1, x_{i}-1\right]
$$

for $i \in 2, \ldots, a+b-1$, and $T_{a+b}=\left[y_{a+b}+1, n\right]$, getting

$$
P=T_{1} \circ S_{1}^{\prime} \circ T_{2} \circ S_{2}^{\prime} \circ \ldots \circ T_{a+b-1} \circ S_{a+b-1}^{\prime} \circ T_{a+b}
$$

where $\circ$ denotes path concatenation. For $i \in\{1, \ldots, a+b\}$, let $\mathcal{T}_{i}=\mathcal{E}\left(P, \mathbf{x}^{\prime}, \operatorname{Id}, V\left(T_{i}\right)\right)$ be the event that all nodes in the subpath $T_{i}$ say "yes" in instance $I^{\prime}$, and let $p_{i}=\operatorname{Pr}\left[\mathcal{T}_{i}\right]$ its probability. Let $J=\left\{j_{1}, \ldots, j_{b}\right\}$ be the set of $b$ indices with maximal values in $\left\{p_{1}, \ldots, p_{a+b}\right\}$. I.e., $p_{j} \geq \max \left\{p_{i} \mid i \in\{1, \ldots, a+b\} \backslash J\right\}$ for every $j \in J$. We are now defining the valid instance $I=(P, \mathbf{x})$ :

$$
\mathbf{x}(v)= \begin{cases}1 & \text { if } v=u_{i} \text { for some } i \in\{1, \ldots, a+b\} \backslash J \\ 0 & \text { otherwise }\end{cases}
$$

We therefore have that $\left\|\mathbf{x}^{\prime}\right\|_{1}=a+b$ and $\|\mathbf{x}\|_{1}=a$, thus $I \in$ AMOS $-a$ while $I^{\prime} \notin$ AMOS $-a$, and both $I, I^{\prime}$ satisfy the promise. We now make the following immediate observation.

Claim A.1 $\operatorname{Pr}\left[\mathcal{E}\left(P, \mathbf{x}, \operatorname{Id}, V\left(T_{i}\right)\right)\right]=p_{i}$, for every $i \notin J$.

This follows since the distance between any two nodes $u$ (resp., $v$ ) in distinct $T_{i}^{\prime} s$ is greater than $t$, which implies that $\mathbf{x}\left(L_{i} \circ T_{i} \circ R_{i}\right)=\mathbf{x}^{\prime}\left(L_{i} \circ T_{i} \circ R_{i}\right)$ where $L_{i}$ (resp., $\left.R_{i}\right)$ is the subpath of length $t$ to the left (resp., to the right) of $T_{i}$ in $P$, from which it follows that under $\mathcal{A}$ the nodes of $T_{i}$ have the same behavior in both instances $I$ and $I^{\prime}$ for every $i \notin J$.

Let $\mathcal{N}$ (resp., $\mathcal{N}^{\prime}$ ) be the event that there exists at least one node in $I$ (resp., $I^{\prime}$ ) that says "no" when applying algorithm $\mathcal{A}$. Similarly, let $\mathcal{Y}$ (resp., $\mathcal{Y}^{\prime}$ ) be the event that all nodes in the configuration $I$ (resp., $I^{\prime}$ ) say "yes". Let $\mathcal{T}=\bigcup_{i=1}^{a+b} \mathcal{T}_{i}$ be the event that all nodes in the subpaths $T_{i}$, for $i \in\{1, \ldots, a+b\}$ say "yes" in the instance $I^{\prime}$. For every $i \in\{1, \ldots, a+b-1\}$, let $\mathcal{S}_{i}=\mathcal{E}\left(P, \mathbf{x}^{\prime}, \operatorname{Id}, V\left(S_{i}^{\prime}\right)\right)$ be the event that all nodes in the $\delta$-secure subpath $S_{i}^{\prime}$ say "yes" in the instance $I^{\prime}$. We have

$$
\operatorname{Pr}(\mathcal{Y})=\operatorname{Pr}[\mathcal{E}(P, \mathbf{x}, \operatorname{Id}, V(P))]
$$


and

$$
\operatorname{Pr}\left(\mathcal{Y}^{\prime}\right)=\operatorname{Pr}\left[\mathcal{E}\left(P, \mathbf{x}^{\prime}, \mathrm{Id}, V(P)\right)\right]
$$

while

$$
\operatorname{Pr}(\mathcal{N})=1-\operatorname{Pr}(\mathcal{Y})
$$

and

$$
\operatorname{Pr}\left(\mathcal{N}^{\prime}\right)=1-\operatorname{Pr}\left(\mathcal{Y}^{\prime}\right) .
$$

Since $\mathcal{A}$ a $(p, q)$-decider, as we assume by contradiction that AMOS- $a \in B_{k}$, we have

$$
\operatorname{Pr}\left(\mathcal{N}^{\prime}\right) \geq q
$$

and thus

$$
\operatorname{Pr}\left(\mathcal{N}^{\prime}\right)>1-p^{1+1 / \widehat{r}+\varepsilon} .
$$

Therefore, $\operatorname{Pr}\left(\mathcal{Y}^{\prime}\right)<p^{1+1 / \widehat{r}+\varepsilon}$. Moreover, since $I \in \operatorname{AMOS}-a$, we also have that $\operatorname{Pr}(\mathcal{Y}) \geq p$. Therefore,

$$
\frac{\operatorname{Pr}\left(\mathcal{Y}^{\prime}\right)}{\operatorname{Pr}(\mathcal{Y})}<p^{1 / \widehat{r}+\varepsilon}
$$

On the other hand, by applying the union bound to the $a+b$ events $\mathcal{T}, \bigcup_{i=1}^{a+b-1} \mathcal{S}_{i}$, we get that

$$
\begin{aligned}
\operatorname{Pr}\left(\mathcal{N}^{\prime}\right) & \leq(1-\operatorname{Pr}[\mathcal{T}])+\sum_{i=1}^{a+b-1}\left(1-\operatorname{Pr}\left[\mathcal{S}_{i}\right]\right) \\
& \leq 1-\left(\prod_{i \notin J} p_{i} \cdot \prod_{j \in J} p_{j}\right)+(a+b-1) \cdot \delta,
\end{aligned}
$$

where the last inequality follows since $S_{i}^{\prime}$ 's are $\delta$-secure subpaths and hence the events $\mathcal{T}_{i}$ 's are independent. We therefore get that

$$
\operatorname{Pr}\left(\mathcal{Y}^{\prime}\right) \geq\left(\prod_{i \notin J} p_{i} \cdot \prod_{j \in J} p_{j}\right)-(a+b-1) \cdot \delta .
$$

Since $\operatorname{Pr}(\mathcal{Y}) \leq \prod_{i \notin J} p_{i}$, it then follows that

$$
\begin{aligned}
\frac{\operatorname{Pr}\left(\mathcal{Y}^{\prime}\right)}{\operatorname{Pr}(\mathcal{Y})} & \geq \frac{\prod_{i \notin J} p_{i} \cdot \prod_{j \in J} p_{j}-(a+b-1) \cdot \delta}{\prod_{i \notin J} p_{i}} \\
& \geq \prod_{j \in J} p_{j}-\frac{(a+b-1) \cdot \delta}{\prod_{i \notin J} p_{i}} .
\end{aligned}
$$

Now, since $I \in$ AMOS $-a$, we have $\prod_{i \notin J} p_{i} \geq \operatorname{Pr}(\mathcal{Y}) \geq p$, and thus

$$
\frac{\operatorname{Pr}\left(\mathcal{Y}^{\prime}\right)}{\operatorname{Pr}(\mathcal{Y})} \geq \prod_{j \in J} p_{j}-\frac{(a+b-1) \cdot \delta}{p} .
$$

Note that

$$
\prod_{j \in J} p_{j} \geq p^{1 / \widehat{r}}
$$


By the definition of $J,|J|=b$, and $\prod_{j \in J} p_{j} \geq p_{i}^{b}$ for every $i \notin J$. In addition, since there are $a$ indices $i \notin J$, we get that $\left(\prod_{j \in J} p_{j}\right)^{a / b} \geq \prod_{i \notin J} p_{i} \geq \operatorname{Pr}(\mathcal{Y}) \geq p$. Combining with the definition of $\widehat{r}$, Eq. A.12, follows. Hence, by Eq. A.11, we get

$$
\operatorname{Pr}\left(\mathcal{Y}^{\prime}\right) / \operatorname{Pr}(\mathcal{Y}) \geq p^{1 / \widehat{r}}-\frac{(a+b-1) \cdot \delta}{p} .
$$

Combining with Eq. A.10 we get that

$$
p^{1 / \widehat{r}}-(a+b-1) \cdot \delta / p<p^{1 / \widehat{r}+\varepsilon},
$$

which is in contradiction to the definition of $\delta$ in Eq. A.9p. We therefore get that AMOS $-a \notin C_{\widehat{r}}(t)$, and since $r \leq \widehat{r}$, it also holds that AMOS $-a \notin C_{r}(t)$ as required. The theorem follows. 


\section{B Figures}

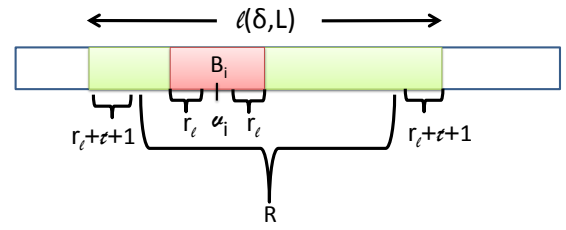

Figure 1: Presented is path $P$. The green $\ell(\delta, \lambda)$-length segment corresponds to $S$. The red subpath $B_{i}$ whose center is $v_{i}$ is a $(\delta, \lambda)$-secure subpath candidate, as $i \in R$.

I'
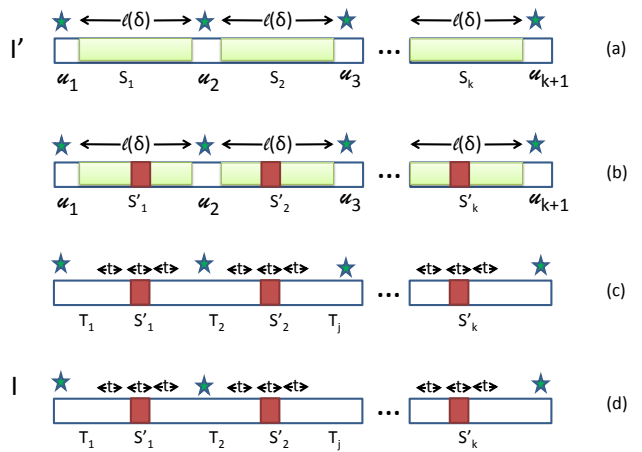

Figure 2: Illustration of the constructions for Theorem 3.1. (a) The instance $I^{\prime}=\left(P, \mathbf{x}^{\prime}\right)$ with $k+1$ leaders separated by $\ell(\delta)$-length segements, $S_{i}$. (b) The $\delta$-secure subpaths $S_{i}^{\prime}$ in each $S_{i}$ are internal to $S_{i}$. (c) The leader-segments $T_{i}$ interleaving with $\delta$-secure subpaths $S_{i}^{\prime}$. (d) The legal instance $I=(P, \mathbf{x})$, the $j^{\text {th }}$ leader of $I^{\prime}$ is discarded, resulting in a $k$ leader instance. 

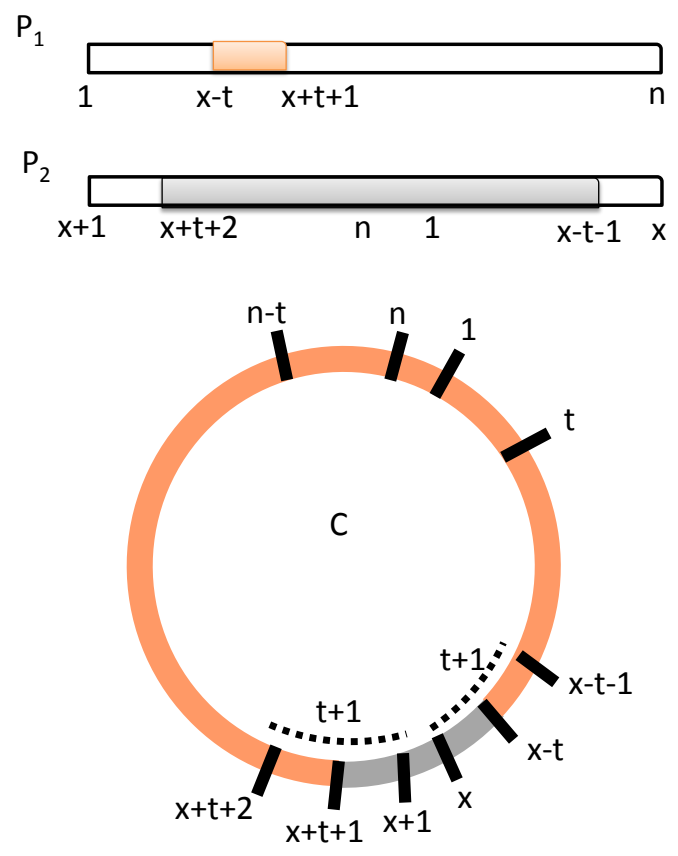

Figure 3: Illustration of the constructions for Theorem 3.3 . Shown are paths $P_{1}, P_{2}$ and cycle $C$. When applying Algorithm $\mathcal{A}$ on path $P_{1}$ (respectively, $P_{2}$ ) and on cycle $C$, the nodes in the segment $[x-t, x+t+1]$ (resp., $[x+t+2, \ldots, 1, \ldots, x+t+1]$ ) behave the same.

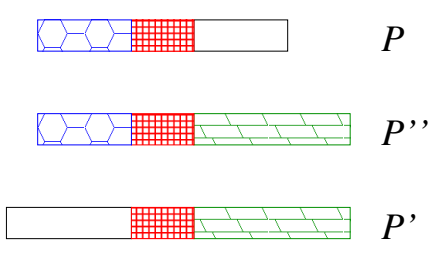

Figure 4: Example of a $\lambda$-path triplet (the red zone is of length at least $\lambda$ ). 


\section{References}

[1] Y. Afek, S. Kutten, and M. Yung. The local detection paradigm and its applications to self stabilization. TCS, 186:199-230, 1997.

[2] N. Alon, L. Babai, and A. Itai. A fast and simple randomized parallel algorithm for the maximal independent set problem. J. Alg., 7:567-583, 1986.

[3] A. Amit, N. Linial, J. Matousek, and E. Rozenman. Random lifts of graphs. In Proc. 12th SODA, 883-894, 2001.

[4] B. Awerbuch, B. Patt-Shamir, and G. Varghese. Self-Stabilization By Local Checking and Correction. Proc. FOCS, 1991, 268-277.

[5] L. Barenboim and M. Elkin. Distributed $(\Delta+1)$-coloring in linear (in delta) time. Proc. 41st STOC, 111-120, 2009.

[6] A. Das Sarma, S. Holzer, L. Kor, A. Korman, D. Nanongkai, G. Pandurangan, D. Peleg and R. Wattenhofer. Distributed Verification and Hardness of Distributed Approximation. Proc. 43rd STOC, 2011.

[7] D Dereniowski and A. Pelc. Drawing maps with advice. JPDC,72:132-143, 2012.

[8] E.W. Dijkstra. Self-stabilization in spite of distributed control. Comm. ACM, 17(11), 643-644, 1974.

[9] S. Dolev, M. Gouda, and M. Schneider. Requirements for silent stabilization. Acta Informatica, 36(6), 447-462, 1999.

[10] P. Fraigniaud, C. Gavoille, D. Ilcinkas and A. Pelc. Distributed Computing with Advice: Information Sensitivity of Graph Coloring. Proc. 34th ICALP, 231-242, 2007.

[11] P. Fraigniaud, D Ilcinkas, and A. Pelc. Communication algorithms with advice. JCSS, 76:222-232, 2008.

[12] P. Fraigniaud, A Korman, and E. Lebhar. Local MST computation with short advice. Proc. 19th SPAA, 154-160, 2007.

[13] P. Fraigniaud, A. Korman, and D. Peleg. Local Distributed Decision. Proc. 52nd FOCS, 708-717, 2011.

[14] P. Fraigniaud and A. Pelc. Decidability Classes for Mobile Agents Computing. Proc. 10th LATIN, 2012.

[15] P. Fraigniaud, S. Rajsbaum, and C. Travers. Locality and Checkability in Wait-free Computing. Proc. 25th DISC, 2011.

[16] P. Fraigniaud, S. Rajsbaum, and C. Travers. Universal Distributed Checkers and Orientation-Detection Tasks. Submitted, 2012.

[17] M. Göös and J. Suomela. Locally checkable proofs. Proc. 30th PODC, 2011.

[18] L. Kor, A. Korman and D. Peleg. Tight Bounds For Distributed MST Verification. Proc. $28 t h$ STACS, 2011.

[19] A. Korman and S. Kutten. Distributed verification of minimum spanning trees. Distributed Computing, 20:253266, 2007.

[20] A. Korman, S. Kutten, and T. Masuzawa. Fast and Compact Self-Stabilizing Verification, Computation, and Fault Detection of an MST. Proc. 30th PODC, 2011.

[21] A. Korman, S. Kutten, and D Peleg. Proof labeling schemes. Distributed Computing, 22:215-233, 2010.

[22] A. Korman, J.S. Sereni, and L. Viennot. Toward More Localized Local Algorithms: Removing Assumptions Concerning Global Knowledge. Proc. 30th PODC, 49-58, 2011.

[23] F. Kuhn. Weak graph colorings: distributed algorithms and applications. Proc. 21st SPAA, 138-144, 2009.

[24] M. Luby. A simple parallel algorithm for the maximal independent set problem. SIAM J. Comput., 15:10361053, 1986.

[25] M. Naor. A Lower Bound on Probabilistic Algorithms for Distributive Ring Coloring. SIAM J. Discrete Math., 4(3): 409-412 (1991). 
[26] M. Naor and L. Stockmeyer. What can be computed locally? SIAM J. Comput. 24(6): 1259-1277 (1995).

[27] A. Panconesi and A. Srinivasan. On the Complexity of Distributed Network Decomposition. J. Alg. 20: 356-374, (1996).

[28] D. Peleg. Distributed Computing: A Locality-Sensitive Approach. SIAM, 2000.

[29] J. Schneider and R. Wattenhofer. A new technique for distributed symmetry breaking. In Proc. 29th PODC, 257-266, 2010. 